Published in final edited form as:

Hum Mutat. 2018 November ; 39(11): 1485-1493. doi:10.1002/humu.23624.

\title{
Assessing the gene-disease association of 19 genes with the RASopathies using the ClinGen gene curation framework
}

\author{
Andrew R. Grant ${ }^{1,{ }^{*}, \text { Brandon J. Cushman }}{ }^{1,{ }^{*}}$, Hélène Cavé ${ }^{2}$, Mitchell W. Dillon ${ }^{3}$, Bruce D. \\ Gelb $^{4}$, Karen W. Gripp ${ }^{5}$, Jennifer A. Lee ${ }^{6}$, Heather Mason-Suares ${ }^{1}$, Katherine A. Rauen ${ }^{7}$, \\ Marco Tartaglia ${ }^{8}$, Lisa $M$. Vincent ${ }^{9}$, and Martin Zenker ${ }^{10}$
}

${ }^{1}$ Laboratory for Molecular Medicine, Partners Healthcare Personalized Medicine, Cambridge, Massachusetts ${ }^{2}$ Département de Génétique, Hôpital Robert Debré and Institut Universitaire d'Hématologie, Université Paris Diderot, Paris-Sorbonne-Cité, Paris, France ${ }^{3}$ Molecular Genetic Testing Laboratory, Icahn School of Medicine at Mount Sinai, New York City, New York ${ }^{4}$ Departments of Pediatrics and Genetic and Genomic Sciences, Mindich Child Health and Development Institute, Icahn School of Medicine at Mount Sinai, New York City, New York ${ }^{5}$ Department of Pediatrics, Nemours/Alfred I. duPont Hospital for Children, Wilmington, Delaware ${ }^{6}$ Molecular Diagnostic Laboratory, Greenwood Genetic Center, Greenwood, South Carolina ${ }^{7}$ Department of Pediatrics, UC Davis Children's Hospital, Sacramento, California ${ }^{8}$ Ospedale Pediatrico Bambino Gesù, Rome, Italy ${ }^{9}$ GeneDx, Gaithersburg, Maryland ${ }^{10}$ Institute of Human Genetics, University Hospital Magdeburg, Magdeburg, Germany

\begin{abstract}
The RASopathies are a complex group of conditions regarding phenotype and genetic etiology. The ClinGen RASopathy Expert Panel (RAS EP) assessed published and other publicly available evidence supporting the association of 19 genes with RASopathy conditions. Using the semiquantitative literature curation method developed by the ClinGen Gene Curation Working Group, evidence for each gene was curated and scored for Noonan syndrome (NS), Costello syndrome, cardiofaciocutaneous syndrome, NS with multiple lentigines, and Noonan-like syndrome with loose anagen hair.

The curated evidence supporting each gene-disease relationship was then discussed and approved by the ClinGen RASopathy Expert Panel. Each association's strength was classified as definitive, strong, moderate, limited, disputed, or no evidence. Eleven genes were classified as definitively associated with at least one RASopathy condition. Two genes classified as strong for association with at least one RASopathy condition while one gene was moderate and three were limited. The
\end{abstract}

Correspondence: Martin Zenker, Institute of Human Genetics, University Hospital Magdeburg, Leipziger Str. 44, 39120 Magdeburg, Germany. martin.zenker@med.ovgu.de.

*Andrew R. Grant and Brandon J. Cushman are co-first authors.

AUTHORSHIP CONTRIBUTIONS

Manuscript was drafted by ARG. Gene validity curation was performed by BJC and ARG and overseen by LMV and MZ. All authors reviewed and approved clinical validity classifications. HC, BDG, KWG, KAR, MT, and MZ provided clinical expertise and differentials for each RASopathy condition. Finalization of clinical validity classifications and manuscript content was overseen by LMV and MZ.

SUPPORTING INFORMATION

Additional supporting information may be found online in the Supporting Information section at the end of the article. 
RAS EP also disputed the association of two genes for all RASopathy conditions. Overall, our results provide a greater understanding of the different gene-disease relationships within the RASopathies and can help in guiding and directing clinicians, patients, and researchers who are identifying variants in individuals with a suspected RASopathy.

\section{Keywords}

ClinGen; genetic; gene curation; genomics; RASopathy

\section{1| INTRODUCTION}

The RASopathies are a collective group of phenotypically related conditions caused by germline pathogenic variants in genes within the Ras/mitogen-activated protein kinase (Ras/ MAPK) signaling pathway. RASopathy conditions, such as Noonan syndrome (NS; MIM\# 163950) and cardiofaciocutaneous (CFC; MIM\# 115150) syndrome, typically present with multiple phenotypic features, including poor growth, cardiac anomalies, ectodermal abnormalities, neurodevelopmental deficits, and increased tumor risk (Nava et al., 2007; Pierpont et al., 2014; Rauen, 2013; Romano et al., 2010; Tidyman \& Rauen, 2009). Most conditions within the RASopathies have been historically described as clinically distinct syndromes and strong correlations have been noted between each individual syndrome and a mutated gene(s) or even a specific allele within a gene. Despite these correlations, these conditions share a considerable amount of overlapping phenotypic features that can complicate clinical diagnoses. Furthermore, variable expressivity has been described in individuals and families sharing the same genotype (Allanson \& Roberts, 1993; Tartaglia et al., 2002). Therefore, the ClinGen RASopathy expert panel (RAS EP) sought to evaluate the current evidence for each gene-condition assertion in order to provide a comprehensive review of Ras/MAPK pathway genes and their causality of a RASopathy condition.

The ClinGen gene curation effort provides formal evidence-based classifications for the association of a gene with a given disease. This type of information is extremely important as clinicians often use molecular testing results to confirm a clinical diagnosis. Therefore, knowing the level of association of a gene with disease can facilitate more accurate clinical diagnoses. This information also allows for more accurate clinical utility and sensitivity in diagnostic laboratory test designs for specific clinical indications. For example, this exercise supports the exclusion of testing for genes with insufficient evidence to support an association with a RASopathy while also informing which RASopathy genes should be included when testing for specific RASopathy conditions (e.g., a CFC panel). While this list of genes does not include the entirety of genes that have been associated with RASopathies to date (e.g., $C B L, N F 1$, and $S P R E D 1$ are not included in this exercise), we curated information for 44 gene-disease associations and confirmed that 56 assertions have not yet been made using published genetic data. Having comprehensive and concise panels is crucial for the practice of molecular medicine, and gene curation has proven to be useful in this endeavor (Strande et al., 2017). 
For certain gene-disease associations, extracting accurate and clear phenotypic information from the literature can be challenging; however, the phenotypic and genetic heterogeneity within the RASopathies is particularly confounding for establishing clear gene associations with specific RASopathy conditions. Due to variable expression and high clinical overlap of the features, clinicians who diagnose RASopathy patients have often used the molecular diagnosis to support a specific phenotypic diagnosis in their patients. For example, if a patient displays phenotypes that overlap between NS and CFC syndrome, they may use the finding of a de novo variant in $M A P 2 K 2$ as supporting evidence that the patient has CFC instead of relying on specific clinical features. While this exemplifies the utility of molecular medicine as a tool for diagnosis, it also highlights the bias for certain genes to be traditionally associated to only certain RASopathy conditions. However, given that the complexities of the pathway have not been studied in enough detail to rule out that certain $M A P 2 K 2$ variants may lead to NS, it is useful to note when evidence supports that a patient with a $M A P 2 K 2$ variant may have NS and not $\mathrm{CFC}$. While a major part of the utility of the gene curation framework lies in acknowledging all potential associations, RASopathy disorders may have distinguishing features that vary either due to the age of clinical presentation and assessment, generalized phenotypic heterogeneity, or even potentially biased ascertainment. Given these phenotypic similarities overall, a patient diagnosed initially with one entity may transition to a different clinical diagnosis over time as different features manifest or clinical diagnoses are refined. Despite this, there are some nuances and notable differences that can be made among these disorders (see Table 1). For example, feature, such as hypertrophic cardiomyopathy is observed across all RASopathies, yet is more commonly observed in patients clinically diagnosed with Costello syndrome (CS; MIM\# 218040) or NS with multiple lentigines (NSML; MIM\# 151100). In general, multiple lentigines are very common, but within the context of a RASopathy they are highly correlated to NSML. Certain hematologic or oncologic manifestations, including solid tumors and transitional cell carcinoma, are highly specific for CS (Table 1). Through systematic collection of published literature and other case-level evidence from diagnostic and research laboratories, these nuances and differences in conditions enable a better understanding of the larger phenotypic spectrum for each gene. The evidence curated by ClinGen can highlight for clinicians which condition(s) have been associated with a gene, and therefore which phenotypic features any given patient may be at risk for. While, we evaluated case evidence in the context of historically well-described, traditional clinical diagnoses that are distinct in the published literature, it was evident that a certain number of cases diverged from these diagnoses when correlated to molecular results. This underscores the need for better nosology in the RASopathy field to refine and reduce diagnostic discrepancies between physicians. Currently, another initiative to tackle this complex issue in conjunction with feedback from the clinical, research, and patient communities is underway.

We curated evidence for the association of 19 genes with five specific RASopathy conditions: NS, CFC, CS, NSML, and Noonan-like syndrome with loose anagen hair (NS/ LAH; MIM\# 607721). To limit the scope and enable for community feedback of this pilot initiative, our gene list primarily consisted of genes typically associated with a gain-offunction (GOF) mechanism leading to a RASopathy in the literature. While many of the 
gene-disease classifications were curated as definitive and historically linked, we also evaluated genes recently linked to RASopathies, for example, PPP1CB and LZTR1, with the aid of additional diagnostic laboratory data publicly available within ClinVar (https:// www.ncbi.nlm.nih.gov/clinvar/). Note, while some phenotype information is provided in ClinVar, additional information was obtained by directly contacting the clinical lab (GeneDx, Gaithersburg, MD). This internal data did not contribute to any divergent classifications, but were found to support the most common disease association for each gene. All divergent assertions evaluated here were found in published literature. We believe that our expert-approved gene-disease validity classifications will be highly informative to patients, researchers, and clinicians interested in the gene-disease associations in the field of RASopathies.

\section{2 | METHODS}

The associations of each of the 19 genes were classified using the ClinGen Gene Curation Standard Operating Procedures (Version 5) to the following specific RASopathy conditions: NS, NS/LAH, CFC, CS, and NSML (Strande et al., 2017). This framework involves a structured evaluation of published literature to produce a semiquantitative score relative to the strength of evidence available for a given gene-disease association. In addition, diagnostic laboratory data publicly available within ClinVar (https://www.ncbi.nlm.nih.gov/ clinvar/) were also assessed for genes with minimal literature. Genes curated in this study include $A 2 M L 1$ (MIM\# 610627), BRAF(MIM\# 164757), HRAS (MIM\# 190020), KRAS (MIM\# 190070), LZTR1 (MIM\# 600574), MAP2K1 (MIM\# 176872), MAP2K2 (MIM\# 601263), MRAS (MIM\# 608435), NRAS (MIM\# 164790), PPP1CB (MIM\# 600590), PTPN11 (MIM\# 176876), RAF1 (MIM\# 164760), RASA1 (MIM\# 139150), RASA2 (MIM\# 601589), RIT1 (MIM\# 609591), RRAS (MIM\# 165090), SHOC2 (MIM\# 602775), SOS1 (MIM\# 182530), and SOS2 (MIM\# 601247). Each association was classified as definitive (12-18 points with replication), strong (12-18 points), moderate (7-11 points), limited (0.1-6 points), no evidence, disputed, or refuted per the ClinGen Gene Curation criteria (Strande et al., 2017).

After primary curation by a ClinGen biocurator, the evidence for each association was presented to clinical RASopathy experts from several different institutions for review. The evidence and the current clinical validity classification and interpretation supporting the gene-disease relationship were discussed at length followed by a vote. If the vote for the classification was unanimous, the association was approved. If the proposed association was unclear or contested, then the association was discussed at length and voting continued until an $80 \%$ quorum from all ClinGen RASopathy Expert Panel (RAS EP) members was achieved. In several cases, the RAS EP approved caveat language for describing and refining the term asserting the clinical validity of an association (Supporting Information, Table S1). For example, some limited associations (i.e., MAP2K2: NS-only one scored published case) were predicted by experts to be eventually disputed/refuted, and the group stressed that viewers of those marked "limited" associations should exercise caution. 


\section{3 | RESULTS}

A total of 19 genes were curated for their associations with five specific RASopathy conditions. The genes found to be definitive for at least one RASopathy condition were BRAF, HRAS, KRAS, MAP2K1, MAP2K2, NRAS, PTPN11, RAF1, RIT1, SHOC2, and $S O S 1$. Clinical presentations of cases in the literature or ClinVar were reviewed for consistency with the clinical diagnoses provided at the time of the report. Most genetic evidence scored was that of de novo variant occurrences. There were no published casecontrol studies to score. All genes historically known to participate within the Ras/MAPK pathway or have homologous function to known genes achieved 0.5 points in the functional score based on the well-established biochemical function and protein interactions of the pathway. Due to the ubiquitous expression of these genes, tissue-specific expression did not provide functional evidence to associate these genes with the RASopathies. Strength of evidence associated with animal models and rescue models was typically downgraded to 0.5 points due to the lack of phenotypic specificity in the models for the discrete RASopathy conditions. Functional alterations from observed variants in patient and nonpatient derived cells were assessed stringently for supporting disease causation and were only scored separately when a distinct assay directly supported a unique mechanism or endpoint for the specific condition being assessed. For example, NSML caused by variation in PTPN11 results in a predicted neomorphic allele that has reduced catalytic activity (but is not equivalent to a null allele, which is known to cause metachondromatosis) compared to the standard increased activity associated with PTPN11-related NS variants (Fragale, Tartaglia, Wu, \& Gelb, 2004; Kontaridis, Swanson, David, Barford, \& Neel, 2006; Noda, Takahashi, Hayashi, Tanuma, \& Hatakeyama, 2016; Oishi, Gaengel et al., 2006; Oishi, Zhang et al., 2009; Yu et al., 2014). Table 2 summarizes the classifications of all curations along with their semiquantitative score for specific RASopathy conditions (maximum out of 18).

\section{4 | DISCUSSION}

\section{1| Gene curation workflow}

While we utilized the ClinGen gene curation framework to assess these associations, there were some unique aspects of our process that should be noted. After primary curation, the scored literature evidence was presented to the ClinGen RAS EP for review. It included, for example, the number of probands with variants in the gene, number of de novo versus familial occurrences, functional data supporting the impact of the variants, and any phenotypic information available. Additionally, any publicly available variant data from diagnostic labs for genes that have been more recently associated with RASopathies, including RRAS, LZTR1, RASA2, A2ML1, RASA1, and MRAS were accessed via ClinVar (see Supporting Information, Table S1). The ClinGen RAS EP evaluated the evidence and commented on whether certain cases and experimental data should be scored. They also commented on the status of the current practices in the field and whether certain genes are traditionally thought of as being associated with certain conditions. In some cases, the ClinGen RAS EP asserted that certain associations may not align with current practices or may be inaccurate relative to the context of a particular case. For example, $B R A F$ alterations are traditionally associated with CFC syndrome; however, $B R A F$ alterations have also been 
identified in patients with features more typical of NS (Lee et al., 2011; Nystrom et al., 2008; Razzaque et al., 2007; Sarkozy et al., 2009; van Trier et al., 2016). In these instances, the RAS EP noted that the age of ascertainment, outdatedness of clinical assessments, and variable expressivity of the RASopathies should be considered when scoring these cases. After thorough discussion of the evidence, the RAS EP voted blindly on the classification of the clinical validity of the association. If a consensus was not achieved, the curation was discussed further until at least $80 \%$ of the RAS EP was in agreement. In these situations, specific language helped in conveying the reasons for the final decision of the RAS EP (Supporting Information, Table S1).

\subsection{Challenges of the curation process}

The RASopathies are a complex group of disorders with substantial overlap across conditions. Additionally, the molecular mechanisms underlying the resultant RASopathy condition are not clearly correlated to specific variation within a gene. Our work highlights which discrete disorders have been shown to be associated with Ras/MAPK genes in the published literature. Unfortunately, a substantial amount of published literature does not provide enough information to differentiate between certain conditions, such as NS versus CFC syndrome. This limitation is particularly related to the fact that most reported cases are children. Young age at presentation is a significant cause of disputable phenotypic classifications. Since the clinicians on the RAS EP could not clinically evaluate the patients described in publications themselves, we were reliant on the diagnosis provided in the literature.

Our decision to maintain separate associations for each gene and specific condition, instead of only providing a clinical validity classification for each gene's association with a general RASopathy, was challenging. While there is an ongoing discussion in the field of the RASopathies as to whether these genes should be classified as a phenotypic spectrum under a broader disease entity, the clinical diagnoses currently provided to patients is historically supported as separate syndromes. Therefore, we felt it was important that our curations investigated each gene's level of evidence for each of the five discrete syndromes. This allowed us to dispute weaker gene-disease associations that lacked convincing evidence. This process underscored the need for clear nosology guidelines for the RASopathies and the clini cians within the ClinGen RAS EP aimed to highlight specific phenotypic features that can assist in the differentiation of the RASopathies (see Table 1).

While we decided that it was important for each curation to assess the clinical validity of the specific conditions, we found that almost the entirety of experimental evidence currently in the literature does not provide differentiated support for a gene's association with discrete conditions. For example, a mouse model with a knock-in $K R A S$ p.Val14Ile variant that has been asserted to be in association with NS displayed short stature, cardiac abnormalities, craniofacial dysmorphisms, splenomegaly, and myeloproliferative disorder, implicating the variant and gene's causal role in development of a RASopathy condition (Hernandez-Porras et al., 2014). However, this mouse model does not provide evidence to discern between NS versus other RASopathies, especially regarding the known phenotypic overlap in humans. There are similar issues with studies showing that variants in these genes cause similar 
functional alterations in the Ras/MAPK pathway. For example, since the impact of $B R A F$ variants associated with CFC syndrome and NS are both assessed by measuring increased phosphorylation of mitogen-activated protein kinase (MEK) or extra-cellular signalregulated kinase (ERK), this evidence cannot be distinctly scored to support the association between $B R A F$ and NS or CFC syndrome. While there are examples of individuals having the same variant and different clinical diagnoses (e.g., p.T241P, p.K499E, andp.E501K), most evidence supports clinical variability is at least to some extent due to variant-specific differences in the phenotypic expression (e.g., $B R A F$ mutations may be associated with more or less severe cognitive impairment) (Sarkozy et al., 2009). This genotype-specific variability of clinical expression likely results in the overlaps in gene-disease associations observed with the RASopathies. For example, an individual with a BRAF mutation and cognitive function in the normal or low-normal range is more likely to be clinically classified as NS.

Since there are several gene-disease associations that are considered uncommon in the field of RASopathies (e.g., the associations of $K R A S$ with Costello and $M A P 2 K 1$ with NS) many of the associations were disputed by our curations. However, if variants in the gene were associated with an "uncommon" disease but identified in a distinct risk profile within the phenotypic spectrum associated with this disease, the RAS EP typically deferred to the calculated classification of limited or moderate evidence, instead of disputing the association. In contrast, if an uncommon disease association was reported for a variant that had repeatedly been observed with the commonly-associated disease for its respective gene, the uncommon association was classified as disputed. This applies, for example, to the associations of CFC syndrome with PTPN11, SHOC2, and RAF1.

\subsection{Examples of challenging curations}

There were several curations in which the lack of clear genotype-phenotype correlations merited careful review in the scoring of genetic evidence to support an association.

Additionally, the identification of the same variant linked to multiple conditions revealed the lack of specificity in molecular evaluations to a given condition, yet still supported a general association to a RASopathy. Some examples are shown below but further details are provided in Supporting Information, Table S1.

4.3.1 KRAS-CFC syndrome- $K R A S$ is traditionally associated with NS, but $K R A S$ variants have also been identified in patients with a clinical diagnosis of CFC. Additionally, there is substantial phenotypic overlap between NS and CFC syndrome. For example, the cardiac abnormalities and facial anomalies possess a very similar spectrum (Table 1). While the severity of intellectual disability, frequency of severe ectodermal anomalies, and presence of neuropathy or other corticospinal tract anomalies can be used to differentiate NS from CFC syndrome, these distinguishing features can still be observed in NS and are age dependent. Therefore, despite sufficient genetic evidence and repetition over time for $K R A S$ and CFC syndrome to establish a definitive classification, these complexities led clinicians in the RAS EP to caution against classifying CFC syndrome as definitively associated with $K R A S$. The RAS EP chose to classify the association as strong. 
With the evidence provided in the literature, it was not always convincing to the RAS EP that a case described as CFC syndrome with a $K R A S$ variant would not develop features more consistent with NS over time due to the fact that many of the patients were less than 10 years of age (Adachi, Abe, Aoki, \& Matsubara, 2012; Niihori et al., 2006; Zenker et al., 2007). Of note, there were cases in the literature who were as old as 26 presenting with features more consistent with CFC syndrome (Sovik et al., 2007). This scenario exemplifies that a molecular diagnosis should not dictate the final clinical diagnosis, but encourage clinicians to scrutinize the phenotypic presentation of the individual over time. While our consistent approach was to accept the diagnoses within the peer-reviewed literature provided and score cases if the variants themselves had sufficient evidence for pathogenicity, in this particular case, the RAS EP decided that the uncertainty surrounding the cases provided exceptional reasons to downgrade the classification from definitive to strong. We cite variant overlap between CFC syndrome and NS patients, variable expressivity, outdated assessments, and young age of ascertainment of diagnoses of affected individuals as reasons for downgrading this classification from definitive to strong (Supporting Information, Table S1).

4.3.2 LZTR1 Associations - LZTR1 is a gene that has been more recently $(<3$ years from first publication) associated with the RASopathies. The published cases to date have presented with features consistent with NS; however, the mechanism of inheritance is variable. Autosomal dominant (AD) variants are exclusively missense (Chen et al., 2014; Yamamoto et al., 2015), while the autosomal recessive (AR) variants are predominantly predicted loss-of-function (LOF) with at least one hypomorphic allele being present in patients with biallelic mutations (Johnston et al., 2018). It could be inferred that biallelic LOF or a GOF, potentially as dominant negative mechanism, missense variant can lead to the Noonan condition. Of note, LZTR1 is not LOF constrained according to Exome Aggregation Consortium (ExAC) data, suggesting that these variants may be tolerated (exac.broadinstitute.org)(Lek et al., 2016). Further identification and publication of LZTR1 RASopathy cases are necessary to further confirm these disease mechanisms. Given that variants in LZTR1 have been shown to segregate with an NS condition in both inheritance patterns and apparently due to different disease mechanisms, we chose to split the curations into scores for both the AR and AD associations of LZTR1 with the RASopathies. Furthermore, since there have only been three publications to date, we utilized publicly available, yet not formally published data from diagnostic laboratories in ClinVar to achieve the most up-to-date evidence score that reflects the current acceptable association in the field. Evidence of multiple confirmed de novo occurrences primarily justified the score of 12 for genetic evidence. Lack of experimental evidence and replication over time prevents a definitive association, thus the $\mathrm{AD}$ association is currently assessed as strong, but is expected to move to definitive in the near future if no contradictory evidence emerges.

4.3.3 BRAF/MAP2K1/MAP2K2: Noonan syndrome-Variants in these genes have repeatedly been associated with NS, but in many publications, the rationale and basis for the clinical diagnosis of NS over CFC is unclear. As mentioned previously, many of the variants associated with NS in these genes have also been reported in association with CFC. This may be further complicated by the lack of consensus criteria and nosology for differentiating 
NS from CFC syndrome clinically. Moreover, functional differences between NS- and CFC syndrome-associated $B R A F / M A P 2 K 1 / M A P 2 K 2$ variants have not been established, so far. These reasons may support categorizing and classifying these genes with a broader disease entity that encompasses both NS and CFC (i.e., a RASopathy), but the RAS EP felt it was necessary to maintain the traditional disease conditions and assess the strength of evidence for each condition associated with these genes in the literature. It should be noted that none of the associations of these genes with NS reached the maximum score for genetic evidence, thus indicating further evaluation of evidence is needed in the future. The RAS EP noted that some variants in $B R A F$ and $M A P 2 K 1$ were consistently associated with NS alone while other variants had broader assertions, indicating the potential for specific genotypephenotype correlations to be elucidated over time with further experimentation and exploration through functional assays. The RAS EP recognized that the NS diagnosis in individuals carrying variants in genes typically associated with CFC syndrome is a major point of discussion when attempting to classify gene-disease associations among RASopathies. The RAS EP asserted that a phenotypic diagnosis based exclusively on the molecular diagnosis from a gene (and not genotype) level in this area would not appropriately reflect the broad phenotypic spectrum associated with mutations in $B R A F$, $M A P 2 K 1$, and MAP2K2.

4.3.4 PTPN11/BRAF/RAF1: NSML-There is a definitive association for PTPN11 variants with NSML. Unlike other associations, there is robust evidence for the major NSML-associated PTPN11 variants (p.Y279C and p.T468M) displaying clearly different functional properties (reduced phosphatase activity) compared to NS-associated variants. However, this has not been shown for all NSML-associated PTPN11 variants (Fragale et al., 2004; Kontaridis et al., 2006; Noda et al., 2016; Oishi, Gaengel et al., 2006; Oishi, Zhang et al., 2009; Yu et al., 2014). Moreover, young individuals lack clear distinguishing phenotypic characteristics between NS and NSML so precise diagnoses may be difficult, thus explaining the reported associations of NS with "classical" NSML-associated variants in the literature. In addition to the distinct functional impact of NSML-associated PTPN11 variants, there is also a risk profile that distinguishes NSML from NS (e.g., high risk of hypertrophic cardiomyopathy (HCM), risk of hearing deficits). The differentiation is less clear for $B R A F$ and $R A F 1$-associations with NSML, and no specific functional impact of NSML-associated $B R A F / R A F 1$ variants has been established in these genes. Furthermore, the RAS EP asserted from their own experience that even the skin phenotype associated with $B R A F$ and $R A F 1$ is distinct from the multiple lentigines phenotype seen in patients with "classical" NSML-related PTPN11 variants.

4.3.5 S SHOC2 with NS/CFC syndrome and CS—Primary curation showed "limited" evidence for an association of $S H O C 2$ with NS, CFC syndrome and CS. After discussion, the RAS EP decided to classify all other disease associations for $S H O C 2$ except NS/LAH as disputed given the primary common pathogenic variant(p.Ser2Gly) in this gene has been reported over 100 times with a clinical diagnosis of NS/LAH. In addition, this mutation has distinct functional consequences, and the resemblance of the condition associated with variants in $P P P 1 C B$, a known direct interaction partner with $S H O C 2$, further supports that NS/LAH is a discrete entity specifically related to alterations in this signaling 
complex module (Aoki, Niihori, Inoue, \& Matsubara, 2016; Rauen, 2013). While it is true that young patients with NS/LAH may have features reminiscent of CS or CFC syndrome (e.g., hair abnormalities, severe feeding issues, and more severe developmental issues than usually seen in NS), the condition evolves to become more Noonan-like in older children. Considering the specific features of NS/LAH, the RAS EP judged that there is insufficient evidence for classifying the diagnosis as CFC syndrome, CS, or NS instead of NS/LAH in any of the published patients. The RAS EP concluded that the reason for divergent associations in the literature is related to the fact that authors were likely only considering the categories NS, CFC syndrome, and CS before NS/LAH existed as a separate clinical entity. Therefore, all the cases that have been presented to date, should be considered under the NS/LAH condition, thus disputing the association of $S H O C 2$ with NS, CFC syndrome, and CS.

\section{5| CONCLUSIONS}

Given the complexities surrounding the RASopathies, we believe that our curation process's thorough assessment of the current published and publicly available evidence in ClinVar provides insight into the current issues in the field. By utilizing the ClinGen gene curation framework and expert review from members of the ClinGen RAS EP, we compiled evidence and commentary on almost 100 RASopathy gene- disease associations. In choosing to assess the evidence for each specific condition for each gene, we provide a comprehensive review of several controversial associations that we believe will be useful in both clinical and molecular diagnoses. Additionally, the difficulties in validating gene-disease associations among RASopathies demonstrate that all authors who publish on genotypephenotype associations in RASopathies should rigorously substantiate rare condition associations with detailed and comprehensive clinical assessments for the purported diagnosis. While more efforts are needed to address clear nosology of these disorders to aid in clinical diagnosis, the possibility of a diagnosis of a definitively-associated condition with a given gene (per the classifications established here) should be extensively scrutinized and essentially ruled out with valid phenotypic evidence before asserting a disease association with only limited or moderate classification.

While we aimed to provide the community with the most up-to-date associations, we caution that these associations are just a snapshot of the evidence currently available. For example, the RAS EP predicts that the nondefinitive associations of newly recognized genes, such as $S O S 2, P P P 1 C B, L Z T R 1$, and $M R A S$ are likely to become definitive over time as sufficient evidence accumulates in the literature. On the other hand, limited associations for genes historically studied are likely to remain limited or even potentially disputed on re-evaluation. As additional evidence becomes available for expert review, the RAS EP will aim to update and refine classifications on the Clinical Genome Gene-Disease Validity website (https:// www.clinicalgenome.org/curation-activities/gene-disease-validity/results/) and we encourage readers to publish any case-level or experimental evidence that may support, challenge, or inform this curation project and its clinical validity classifications. 


\title{
Supplementary Material
}

Refer to Web version on PubMed Central for supplementary material.

\section{ACKNOWLEDGMENT}

\begin{abstract}
Research reported in this publication was supported by the National Human Genome Research Institute (NHGRI) under award number U41HG006834. MZ received support from German Federal Ministry of Education and Research (BMBF): NSEuroNet (FKZ 01GM1602A), German Ministry of Education and Reserach (BMBF): GeNeRARe (FKZ 01GM1519A).
\end{abstract}

The authors would like to thank Heidi Rehm, Jonathan Berg, Laura Milko, Courtney Thaxton, Erin Riggs, Marina DiStefano, and the additional members of the ClinGen Gene Curation Working Group and Clinical Domain Oversight Committee for their points of feedback on the gene curation approach and manuscript.

Funding information

National Human Genome Research Institute, Grant/Award Number: U41HG006834; German Federal Ministry of Education and Research (BMBF): NSEuroNet, Grant/Award Number: FKZ 01GM1602A; German Ministry of Education and Reserach (BMBF): GeNeRARe, Grant/Award Number: FKZ 01GM1519A

\section{REFERENCES}

Adachi M, Abe Y, Aoki Y, \& Matsubara Y (2012). Epilepsy in RAS/MAPK syndrome: Two cases of cardio-facio-cutaneous syndrome with epileptic encephalopathy and a literature review. Seizure: The Journal of the British Epilepsy Association, 21(1), 55-60. 10.1016/j.seizure.2011.07.013

Allanson JE, \& Roberts AE (1993). Noonan syndrome In Adam MP,Ardinger HH, Pagon RA, Wallace SE, Bean LJH, Stephens K, \& Amemiya A (Eds.), GeneReviews (R). Seattle, WA: University of Washington

Aoki Y, Niihori T, Inoue S, \& Matsubara Y (2016). Recent advances in RASopathies. Journal of Human Genetics, 61(1), 33-39.10.1038/jhg.2015.114 [PubMed: 26446362]

Chen PC, Yin J, Yu HW, Yuan T, Fernandez M, Yung CK, ... Kucherlapati R (2014). Next-generation sequencing identifies rare variants associated with Noonan syndrome. Proceedings of National Academy Science of the United States of America, 111(31), 11473-11478. 10.1073/pnas. 1324128111

Fragale A, Tartaglia M, Wu J, \& Gelb BD (2004). Noonan syndrome-associated SHP2/PTPN11 mutants cause EGF-dependent prolonged GAB1 binding and sustained ERK2/MAPK1 activation. Human Mutation, 23(3), 267-277. 10.1002/humu.20005 [PubMed: 14974085]

Gelb BD, \& Tartaglia M (1993). Oonan syndrome with multiple lentigines In Adam MP, Ardinger HH, Pagon RA, Wallace SE, Bean LJH, Stephens K, \& Amemiya A (Eds.), GeneReviews (R). Seattle, WA: University of Washington.

Gripp KW, \& Lin AE (1993). Costello syndrome In Adam MP, Ardinger HH, Pagon RA, Wallace SE, Bean LJH, Stephens K, \& Amemiya A (Eds.), GeneReviews (R). Seattle, WA: University of Washington.

Hernandez-Porras I, Fabbiano S, Schuhmacher AJ, Aicher A, Canamero M, Camara JA, ... Barbacid M (2014). K-RasV14I recapitulates Noonan syndrome in mice. Proceedings of National Academy Science of the United States of America, 111(46), 16395-16400. 10.1073/pnas.1418126111

Johnston JJ, van der Smagt JJ, Rosenfeld JA, Pagnamenta AT, Alswaid A, Baker EH, ... Biesecker LG (2018). Autosomal recessive Noonan syndrome associated with biallelic LZTR1 variants. Genetics in Medicine. 10.1038/gim.2017.249

Kontaridis MI, Swanson KD, David FS, Barford D, \& Neel BG (2006). PTPN11 (Shp2) mutations in LEOPARD syndrome have dominant negative, not activating, effects. Journal of Biological Chemistry, 281(10), 6785-6792. 10.1074/jbc.M513068200 [PubMed: 16377799]

Lee BH, Kim JM, Jin HY, Kim GH, Choi JH, \& Yoo HW (2011). Spectrum of mutations in Noonan syndrome and their correlation with phenotypes. Journal of Pediatrics, 159(6), 1029-1035. 10.1016/j.jpeds.2011.05.024 [PubMed: 21784453] 
Lek M, Karczewski KJ, Minikel EV, Samocha KE, Banks E, Fennell T, ... Exome Aggregation Consortium. (2016). Analysis of protein-coding genetic variation in 60,706 humans. Nature, 536(7616), 285-291. 10.1038/nature19057 [PubMed: 27535533]

Nava C, Hanna N, Michot C, Pereira S, Pouvreau N, Niihori T, ... Cave H (2007). Cardio-faciocutaneous and Noonan syndromes due to mutations in the RAS/MAPK signalling pathway: Genotype-phenotype relationships and overlap with Costello syndrome. Journal of Medical Genetics, 44(12), 763-771. 10.1136/jmg.2007.050450 [PubMed: 17704260]

Niihori T, Aoki Y, Narumi Y, Neri G, Cave H, Verloes A, ... Matsubara Y (2006). Germline KRAS and BRAF mutations in cardio-facio-cutaneous syndrome. Nature Genetics, 38(3), 294-296. 10.1038/ng1749 [PubMed: 16474404]

Noda S, Takahashi A, Hayashi T, Tanuma S, \& Hatakeyama M (2016). Determination of the catalytic activity of LEOPARD syndrome-associated SHP2 mutants toward parafibromin, a bona fide SHP2 substrate involved in Wnt signaling. Biochemical and Biophysical Research Communications, 469(4), 1133-1139. 10.1016/j.bbrc.2015.12.117 [PubMed: 26742426]

Nystrom AM, Ekvall S, Berglund E, Bjorkqvist M, Braathen G, Duchen K, ... Bondeson ML (2008). Noonan and cardio-facio-cutaneous syndromes: Two clinically and genetically overlapping disorders. Journal of Medical Genetics, 45(8), 500-506. 10.1136/jmg.2008.057653 [PubMed: 18456719]

Oishi K, Gaengel K, Krishnamoorthy S, Kamiya K, Kim IK, Ying H, ... Gelb BD (2006). Transgenic Drosophila models of Noonan syndrome causing PTPN11 gain-of-function mutations. Human Molecular Genetics, 15(4), 543-553. 10.1093/hmg/ddi471 [PubMed: 16399795]

Oishi K, Zhang H, Gault WJ, Wang CJ, Tan CC, Kim IK, ... Gelb BD (2009). Phosphatase-defective LEOPARD syndrome mutations in PTPN11 gene have gain-of-function effects during Drosophila development. Human Molecular Genetics, 18(1), 193-201. 10.1093/hmg/ddn336 [PubMed: 18849586]

Pierpont ME, Magoulas PL, Adi S, Kavamura MI, Neri G, Noonan J, ... Rauen KA (2014). Cardiofacio-cutaneous syndrome: Clinical features, diagnosis, and management guidelines. Pediatrics, 134(4), e1149-e1162. 10.1542/peds.2013-3189 [PubMed: 25180280]

Rauen KA (1993). Cardiofaciocutaneous syndrome In Adam MP, Ardinger HH, Pagon RA, Wallace SE, Bean LJH, Stephens K, \& Amemiya A (Eds.), GeneReviews (R). Seattle, WA: University of Washington.

Rauen KA (2013). The RASopathies. Annual Review of Genomics and Human Genetics, 14, 355-369. 10.1146/annurev-genom-091212-153523

Razzaque MA, Nishizawa T, Komoike Y, Yagi H, Furutani M, Amo R, ... Matsuoka R (2007). Germline gain-of-function mutations in RAF1 cause Noonan syndrome. Nature Genetics, 39(8), 1013-1017. 10.1038/ng2078 [PubMed: 17603482]

Roberts AE, Allanson JE, Tartaglia M, \& Gelb BD (2013). Noonan syndrome. Lancet, 381(9863), 333-342. 10.1016/S0140-6736(12)61023-X [PubMed: 23312968]

Romano AA, Allanson JE, Dahlgren J, Gelb BD, Hall B, Pierpont ME, ... Noonan JA (2010). Noonan syndrome: Clinical features, diagnosis, and management guidelines. Pediatrics, 126(4), 746-759. 10.1542/peds.2009-3207 [PubMed: 20876176]

Sarkozy A, Carta C, Moretti S, Zampino G, Digilio MC, Pantaleoni F, ... Tartaglia M (2009). Germline BRAF mutations in Noonan, LEOPARD, and cardiofaciocutaneous syndromes: Molecular diversity and associated phenotypic spectrum. Human Mutation, 30(4), 695-702. 10.1002/humu.20955 [PubMed: 19206169]

Sovik O, Schubbert S, Houge G, Steine SJ, Norgard G, Engelsen B, ... Molven A (2007). De novo HRAS and KRAS mutations in two siblings with short stature and neuro-cardio-facio-cutaneous features. Journal of Medical Genetics, 44(7), e84 10.1136/jmg.2007.049361 [PubMed: 17601930]

Strande NT, Riggs ER, Buchanan AH, Ceyhan-Birsoy O, DiStefano M, Dwight SS, ... Berg JS (2017). Evaluating the clinical validity of gene-disease associations: An evidence-based framework developed by the clinical genome resource. American Journal of Human Genetics, 100(6), 895906. 10.1016/j.ajhg.2017.04.015 [PubMed: 28552198]

Tartaglia M, Kalidas K, Shaw A, Song X, Musat DL, van der Burgt I, ... Gelb BD (2002). PTPN11 mutations in Noonan syndrome: Molecular spectrum, genotype-phenotype correlation, and 
phenotypic heterogeneity. American Journal of Human Genetics, 70(6), 1555-1563. 10.1086/340847 [PubMed: 11992261]

Tidyman WE, \& Rauen KA (2009). The RASopathies: Developmental syndromes of Ras/MAPK pathway dysregulation. Current Opinion in Genetics and Development, 19(3), 230-236. 10.1016/ j.gde.2009.04.001 [PubMed: 19467855]

van Trier DC, Vos AM, Draaijer RW, van der Burgt I, Draaisma JM, \& Cruysberg JR (2016). Ocular manifestations of Noonan syndrome: A prospective clinical and genetic study of 25 patients. Ophthalmology, 123(10), 2137-2146. 10.1016/j.ophtha.2016.06.061 [PubMed: 27521173]

Yamamoto GL, Aguena M, Gos M, Hung C, Pilch J, Fahiminiya S, ... Bertola DR (2015). Rare variants in SOS2 and LZTR1 are associated with Noonan syndrome. Journal of Medical Genetics, 52(6), 413-421. 10.1136/jmedgenet-2015-103018 [PubMed: 25795793]

Yu ZH, Zhang RY, Walls CD, Chen L, Zhang S, Wu L, ... Zhang ZY (2014). Molecular basis of gainof-function LEOPARD syndrome-associated SHP2 mutations. Biochemistry, 53(25), 4136-4151. 10.1021/bi5002695 [PubMed: 24935154]

Zenker M, Lehmann K, Schulz AL, Barth H, Hansmann D, Koenig R, ... Kutsche K (2007).

Expansion of the genotypic and phenotypic spectrum in patients with KRAS germline mutations. Journal of Medical Genetics, 44(2), 131-135. 10.1136/jmg.2006.046300 [PubMed: 17056636] 


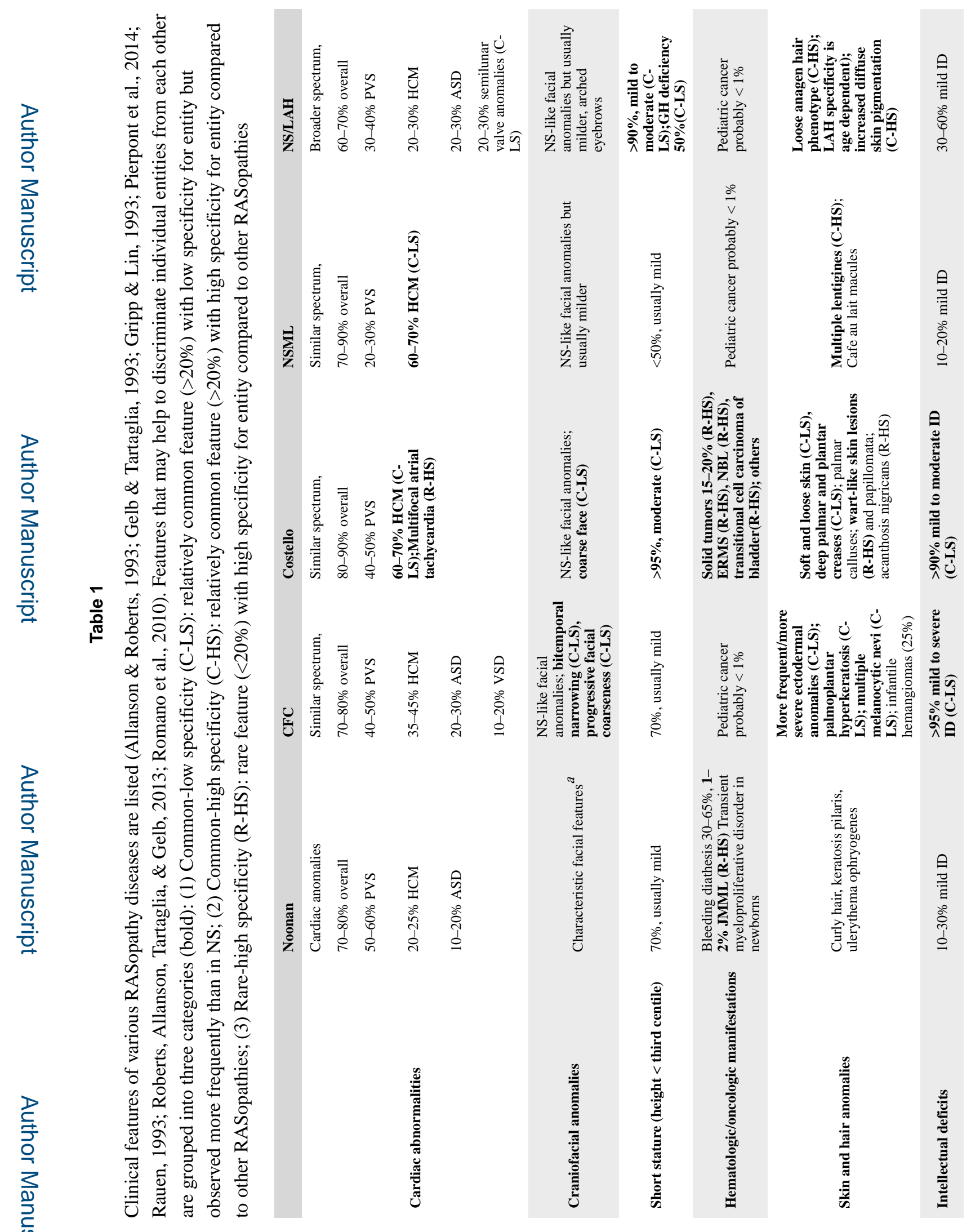

Hum Mutat. Author manuscript; available in PMC 2019 November 01. 
Grant et al.

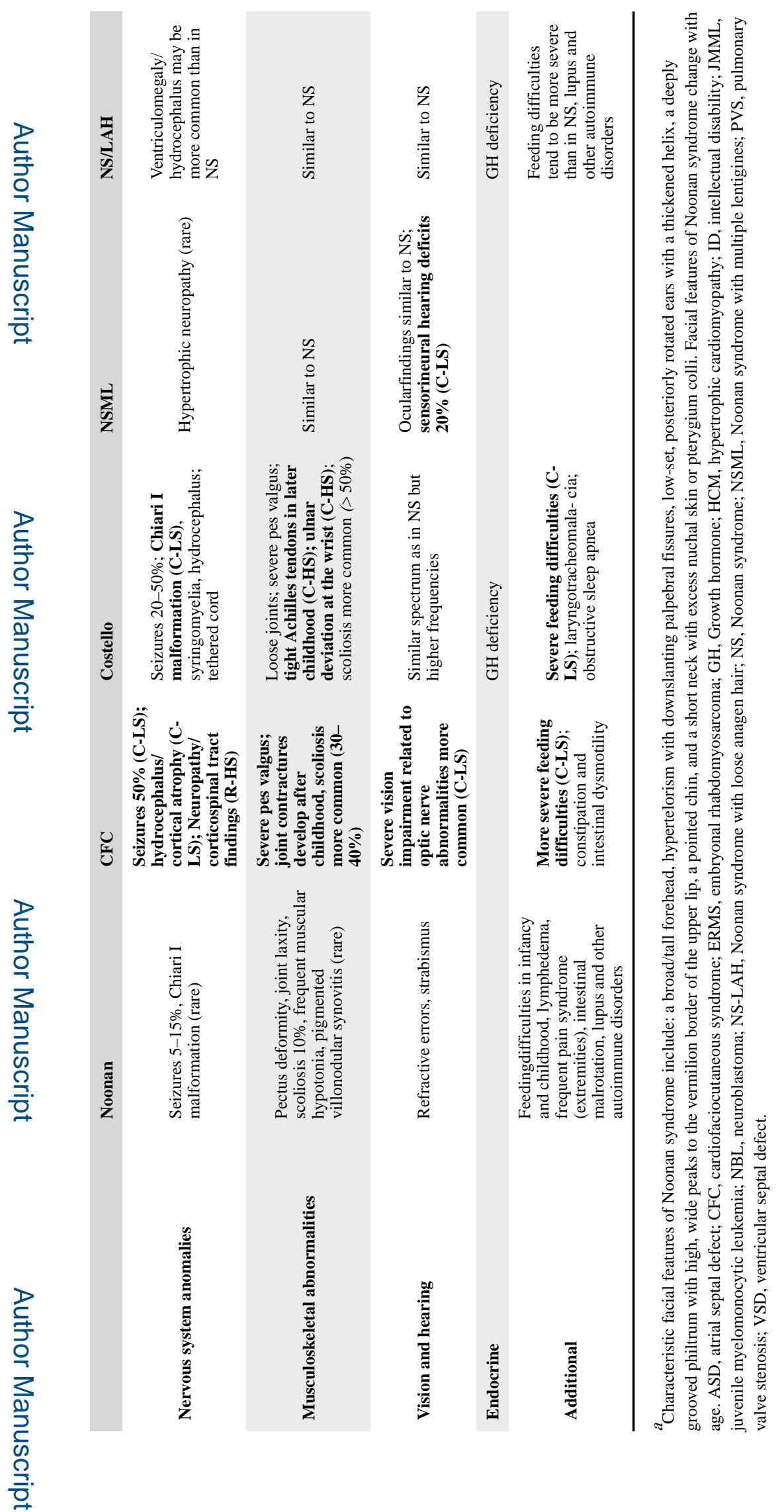

Hum Mutat. Author manuscript; available in PMC 2019 November 01. 


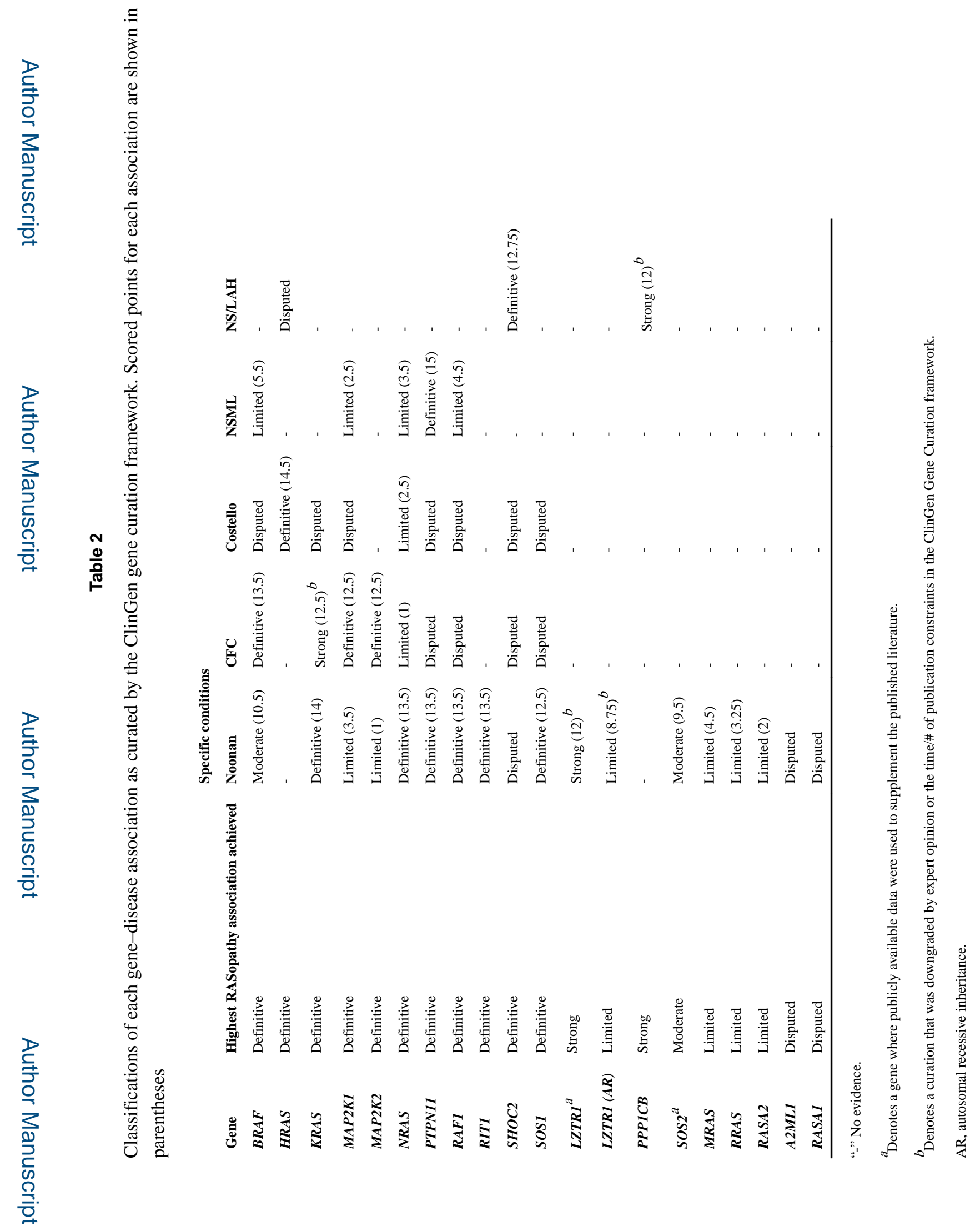

Hum Mutat. Author manuscript; available in PMC 2019 November 01. 